$$
\text { for }
$$

\title{
Solvent-Minimized Synthesis of 4CzIPN and Related Organic Fluorophores via Ball Milling
}

\author{
Jamie A. Leitch,* Harry R. Smallman, Duncan L. Browne* \\ Department of Pharmaceutical and Biological Chemistry, UCL (University College London), 29- \\ 39 Brunswick Square, Bloomsbury, London, W1CN 1AX, United Kingdom \\ *j.leitch@ucl.ac.uk, *duncan.browne@ucl.ac.uk
}

\section{Contents}

1. Mechanochemistry Equipment

2. NMR Spectra. 


\section{Mechanochemistry Equipment}

Mixer mills: Small-scale mechanochemical reactions $(0.5 \mathrm{mmol})$ were conducted using an Insolido Tech IST636 mixer mill (below, see www.insolidotech.org for more details)
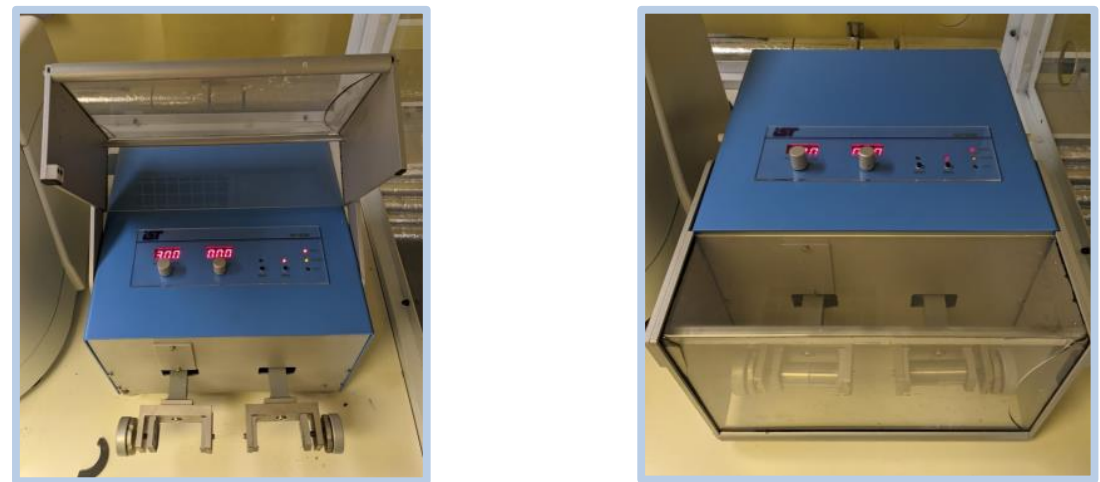

Large-scale mechanochemical reactions $(2.5 \mathrm{mmol})$ were conducted using a Retsch MM400 mixer mill (below, see www.retsch.com for more details)
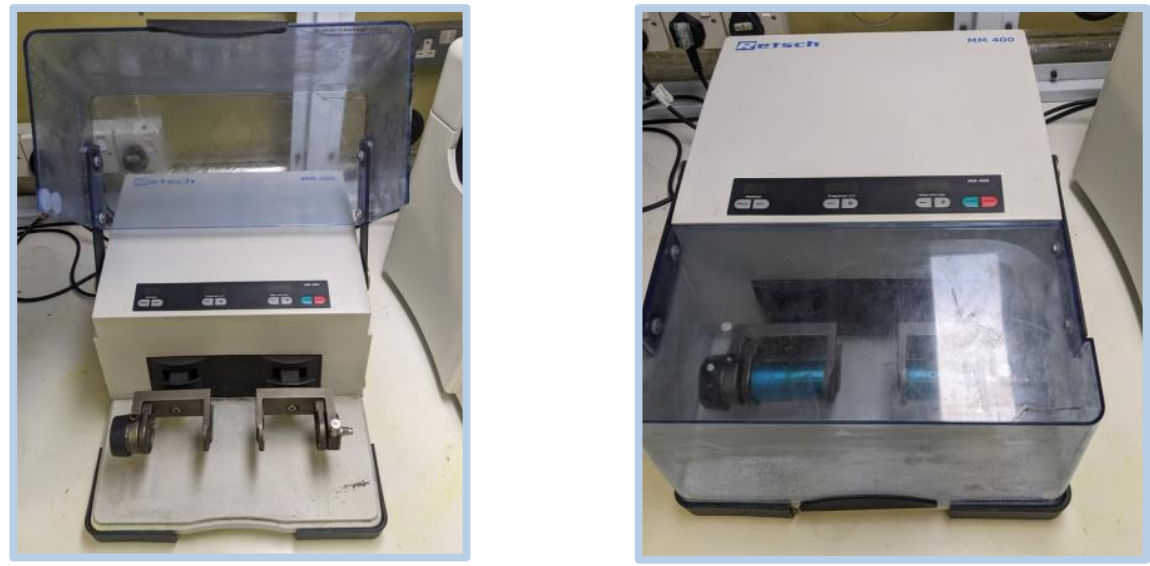

Jars: Mechanochemical reactions were conducted using Formtech Scientific SMARTSNAPTM stainless steel grinding jars with Teflon seals (for further details see ww.formtechscientific.com) at either $30 \mathrm{~mL}$ (left, for $2.5 \mathrm{mmol}$ reaction) or $15 \mathrm{~mL}$ (right, for $0.5 \mathrm{mmol}$ reactions).

Balls: Stainless steel balls were purchased from Bearing Boys limited (www.bearingboys.co.uk) and either

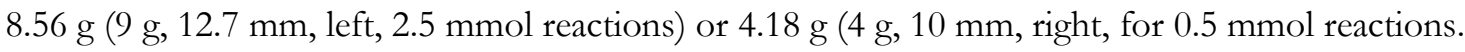

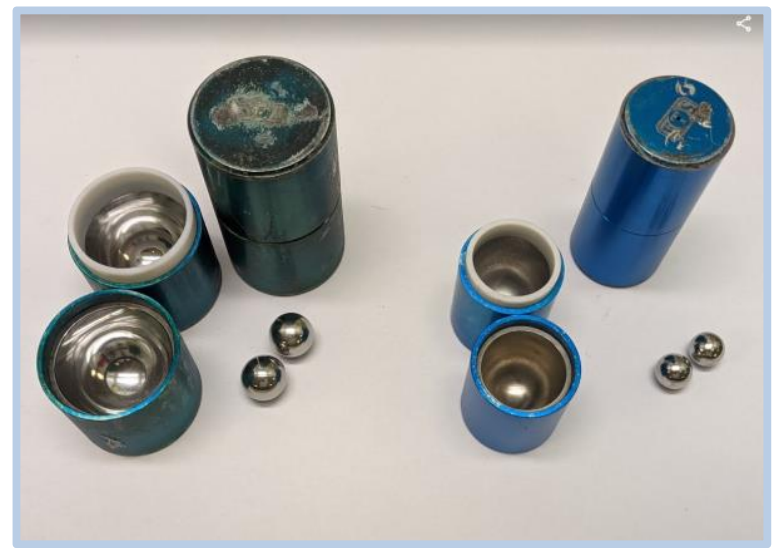




\section{NMR Spectra}

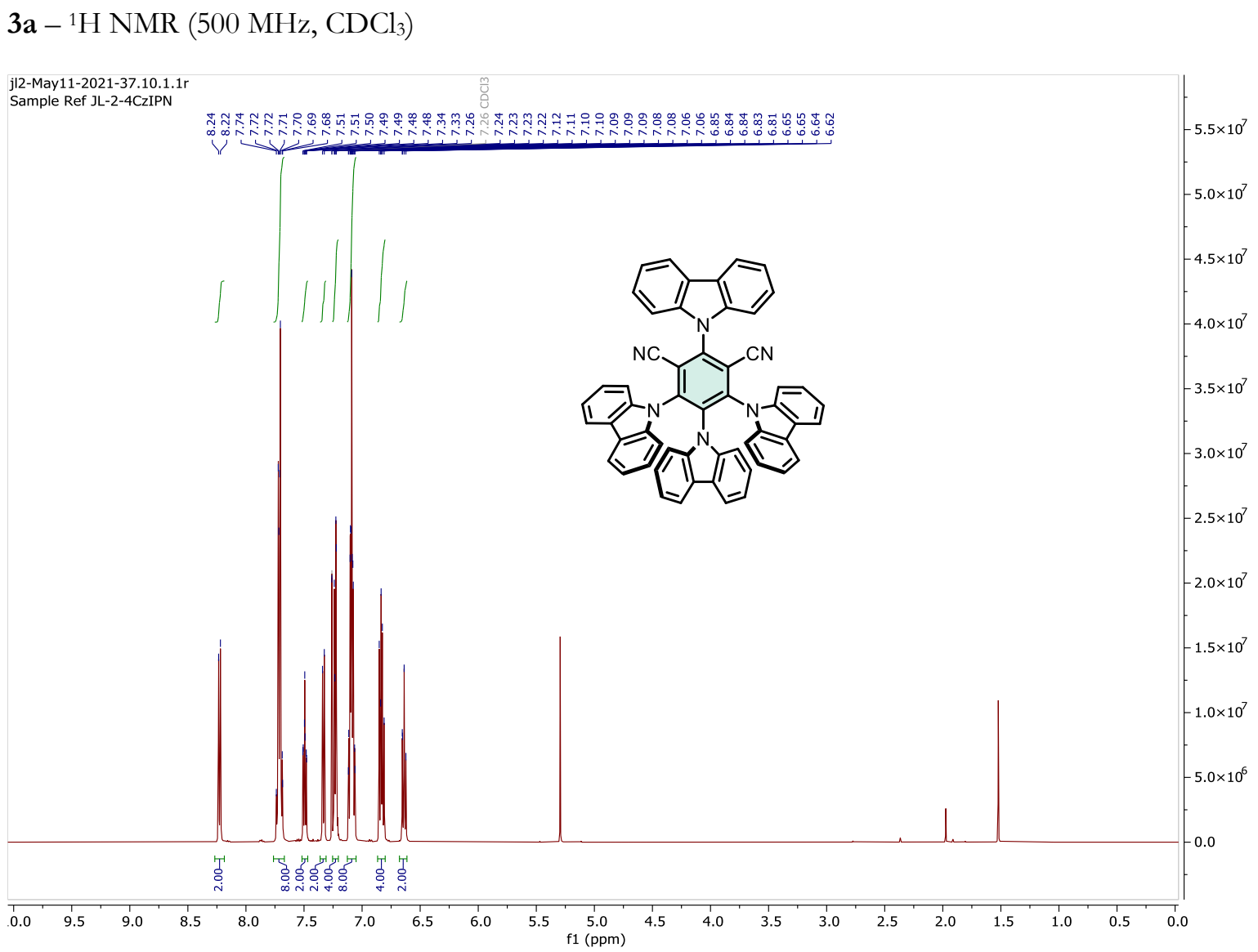

$3 \mathbf{a}-{ }^{13} \mathrm{C}\left\{{ }^{1} \mathrm{H}\right\}$ NMR $\left(126 \mathrm{MHz}, \mathrm{CDCl}_{3}\right)$

j12-May04-2021-55.11.1.1r

Sample Ref JL-2-4CZIPN-Scale-Up
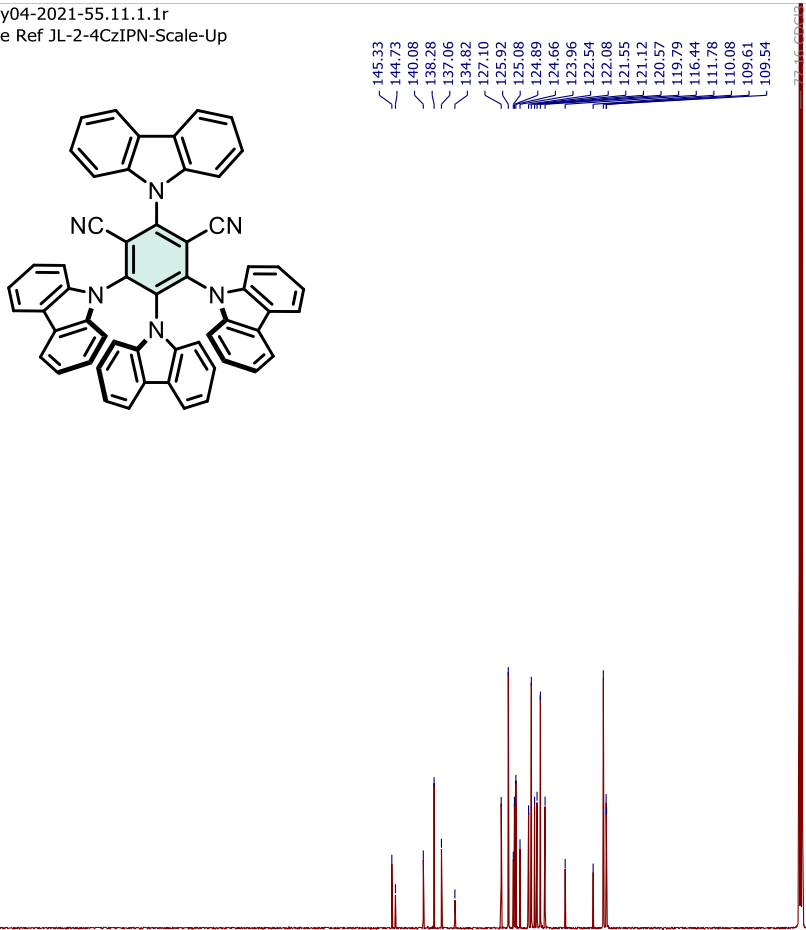

$-2.1 \times 10^{9}$

$2.0 \times 10^{9}$

$-1.9 \times 10^{9}$

$-1.8 \times 10^{9}$

$-1.7 \times 10^{9}$

$1.6 \times 10^{9}$

$-1.5 \times 10^{9}$

$-1.4 \times 10^{9}$

$-1.3 \times 10^{9}$

$-1.2 \times 10^{9}$

$-1.1 \times 10^{9}$

$-1.0 \times 10^{9}$

$-9.0 \times 10^{8}$

$-8.0 \times 10^{8}$

$-7.0 \times 10^{8}$

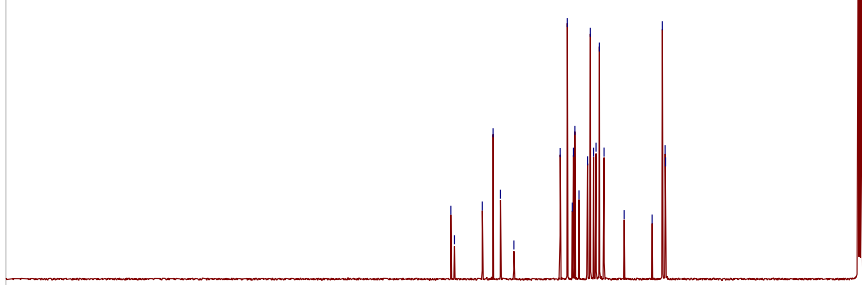

$-6.0 \times 10^{8}$

$-5.0 \times 10^{8}$

$-4.0 \times 10^{8}$

$-3.0 \times 10^{8}$ 
$3 \mathbf{b}-{ }^{1} \mathrm{H}$ NMR $\left(500 \mathrm{MHz}, \mathrm{DMSO}-d_{6}\right)$

j12-Apr13-2021-8.10.1.1

Sample Ref JL-2-019-A-DMSO

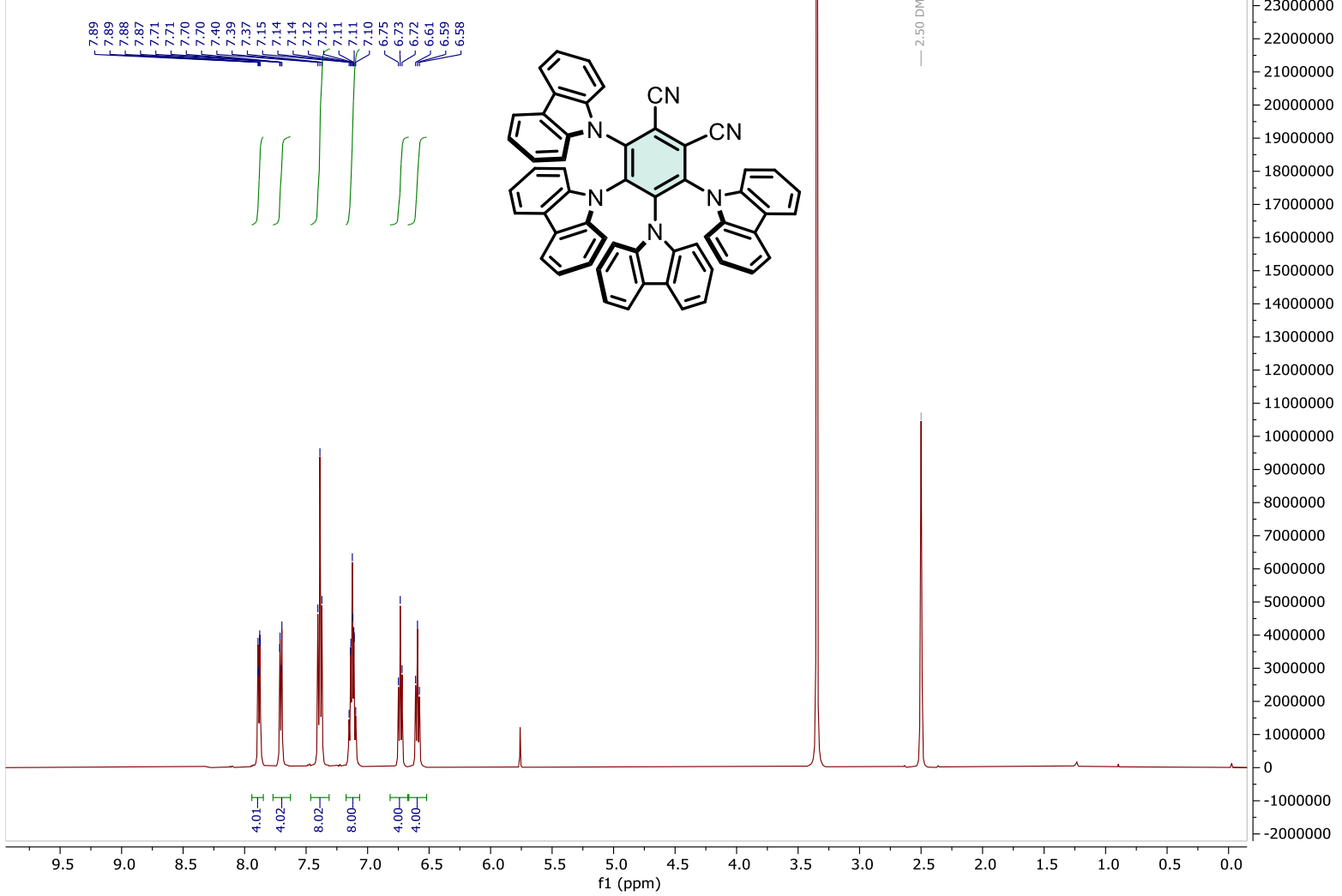

$3 \mathbf{b}-{ }^{13} \mathrm{C}\left\{{ }^{1} \mathrm{H}\right\}$ NMR $\left(126 \mathrm{MHz}\right.$, DMSO- $\left.d_{6}\right)$

j12-Apr13-2021-8.11.1.1 Sample Ref JL-2-019-A-DMSO

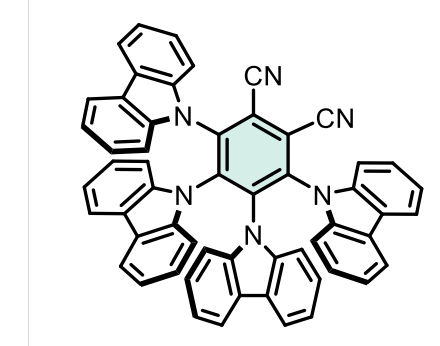

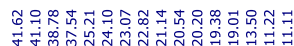

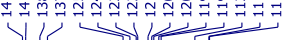

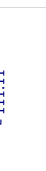


$3 \mathbf{c}-{ }^{1} \mathrm{H}$ NMR $\left(500 \mathrm{MHz}, \mathrm{CD}_{2} \mathrm{Cl}_{2}\right)$

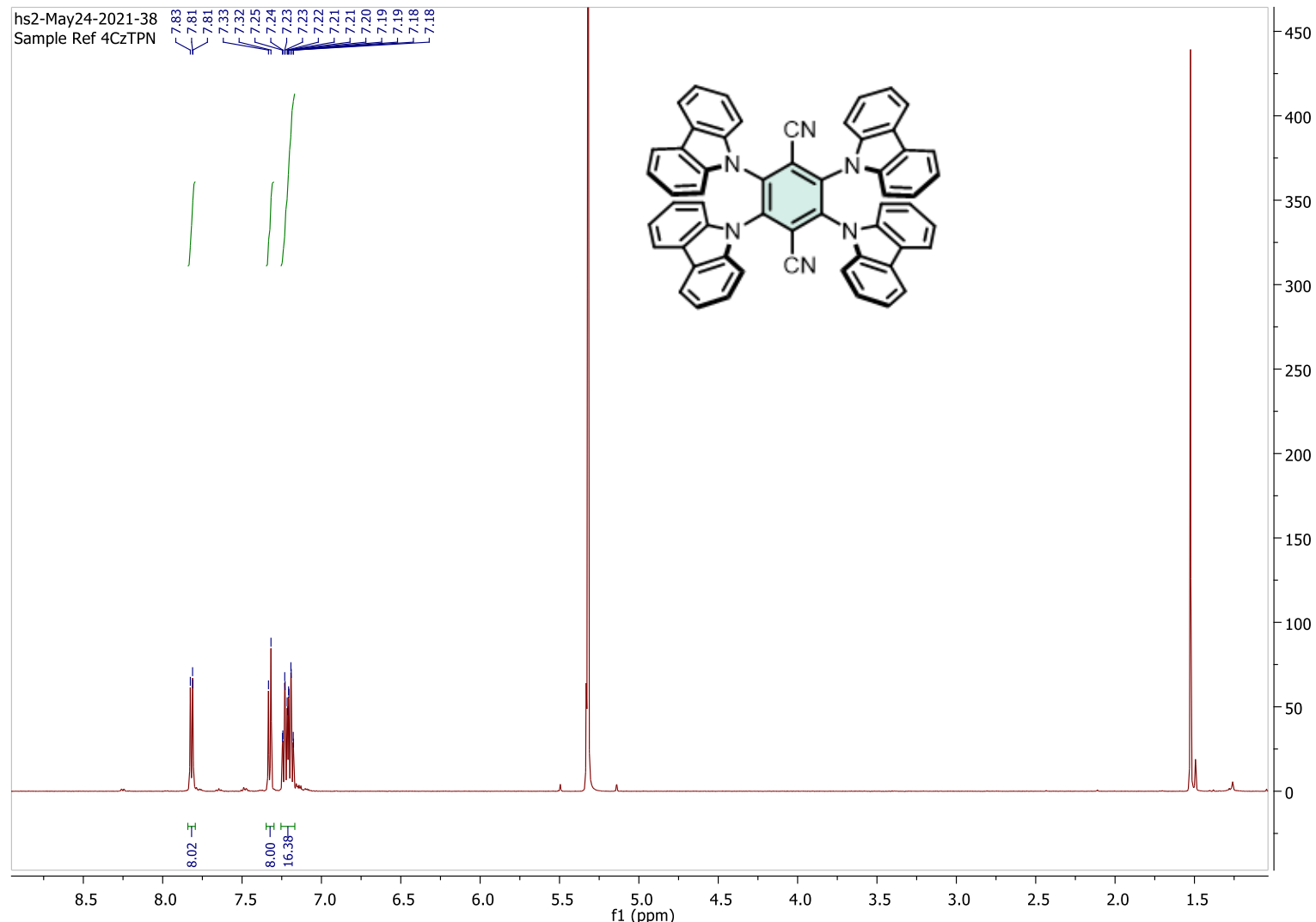

$3 \mathbf{c}-{ }^{13} \mathrm{C}\left\{{ }^{1} \mathrm{H}\right\}$ NMR $\left(126 \mathrm{MHz}, \mathrm{CD}_{2} \mathrm{Cl}_{2}\right)$

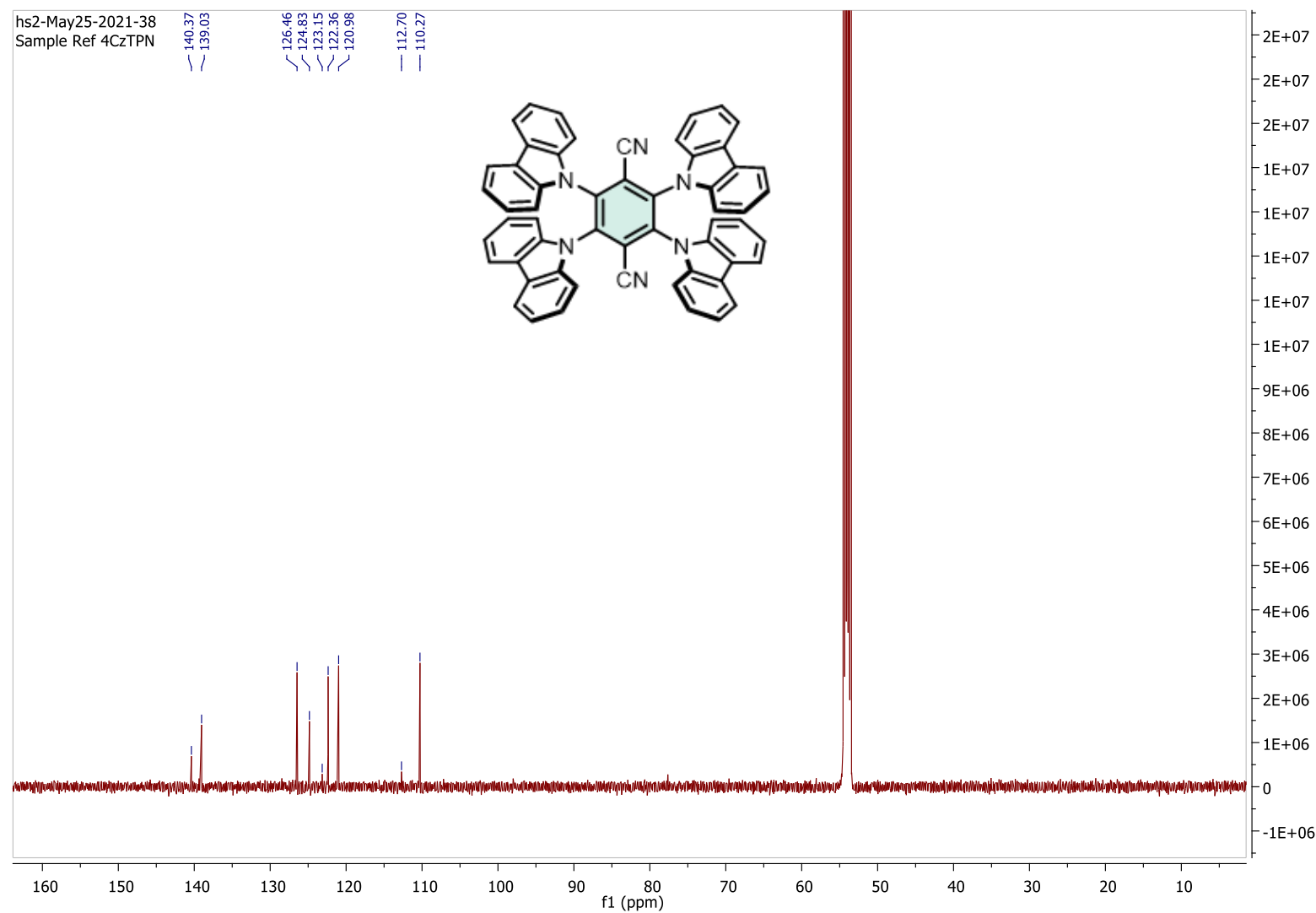


3d - ${ }^{1} \mathrm{H}$ NMR $\left(500 \mathrm{MHz}, \mathrm{CD}_{2} \mathrm{Cl}_{2}\right)$

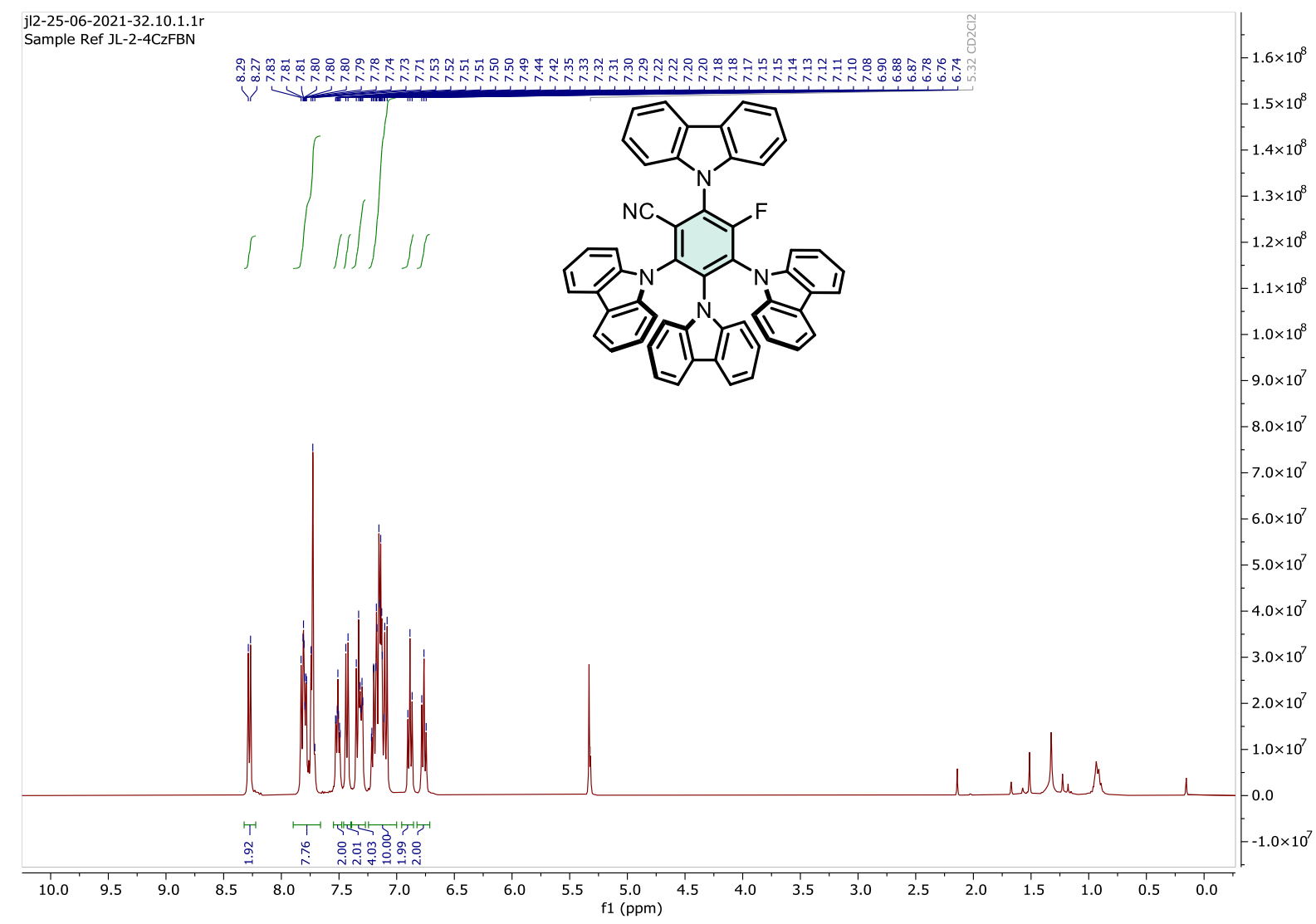

3d $-{ }^{13} \mathrm{C}\left\{{ }^{1} \mathrm{H}\right\}$ NMR $\left(100 \mathrm{MHz}, \mathrm{CD}_{2} \mathrm{Cl}_{2}\right)$

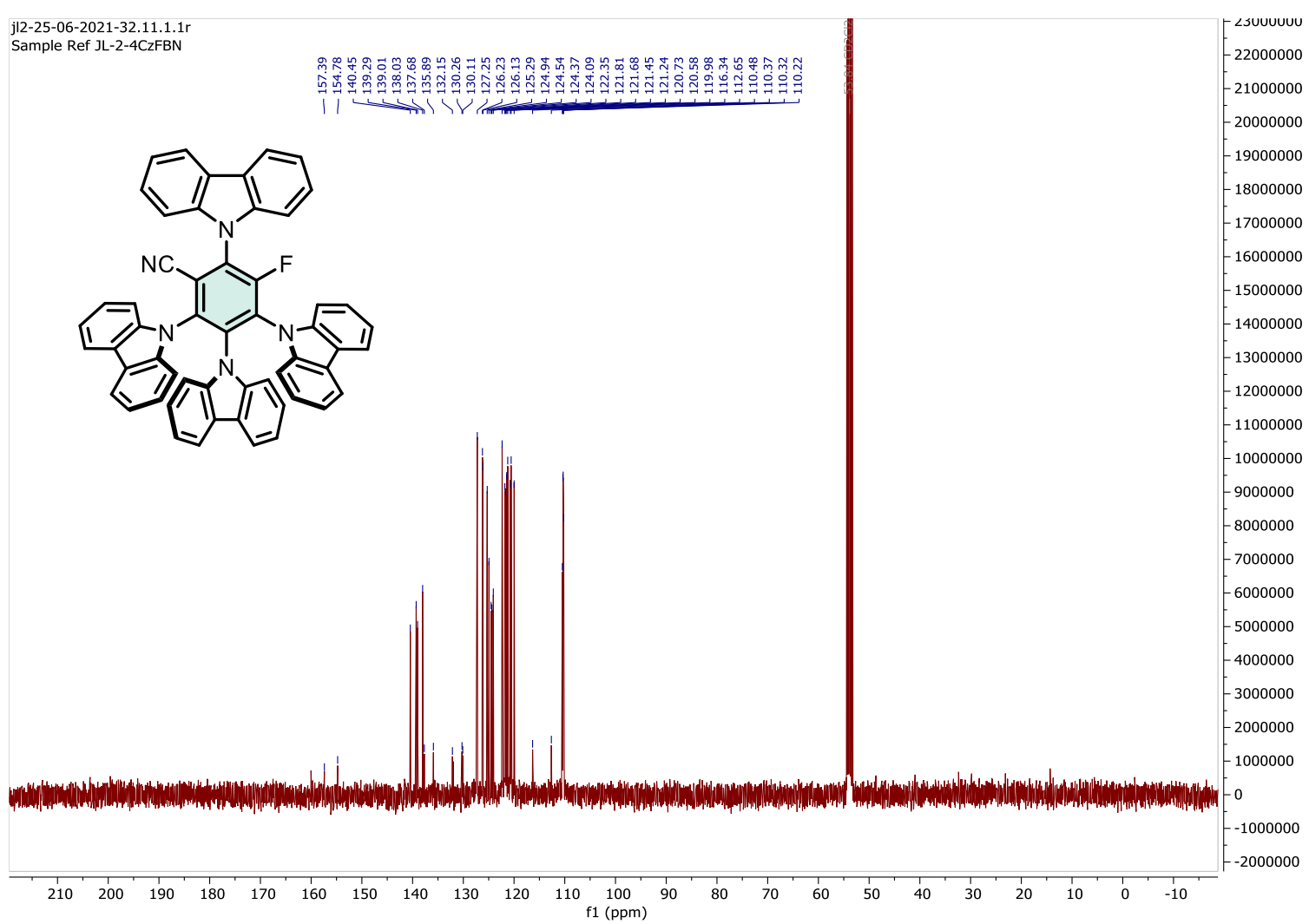


$3 \mathbf{e}-{ }^{-1} \mathrm{H}$ NMR $\left(500 \mathrm{MHz}, \mathrm{CDCl}_{3}\right)$

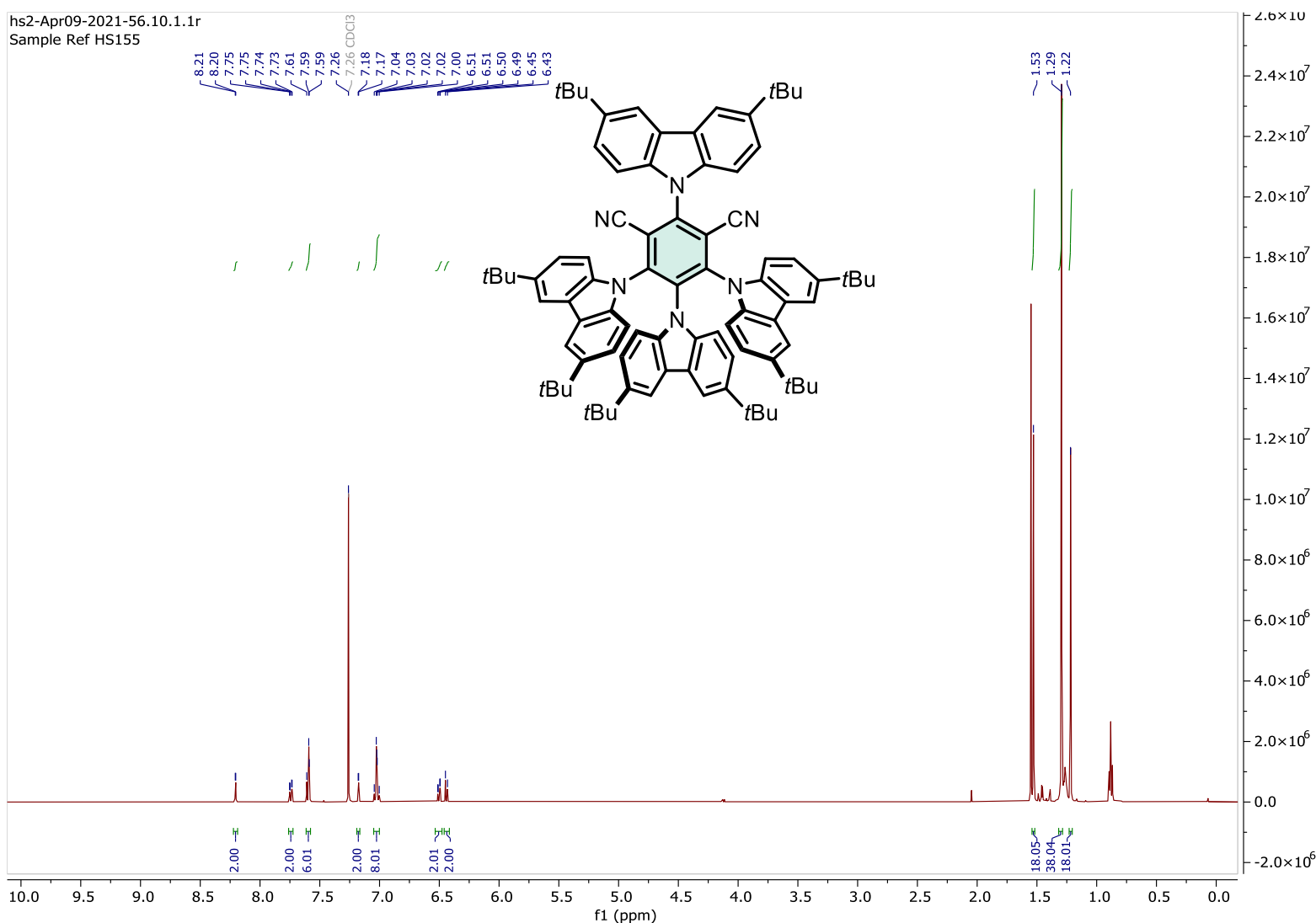

$3 \mathbf{e}-{ }^{13} \mathrm{C}\left\{{ }^{1} \mathrm{H}\right\} \mathrm{NMR}\left(126 \mathrm{MHz}, \mathrm{CDCl}_{3}\right)$

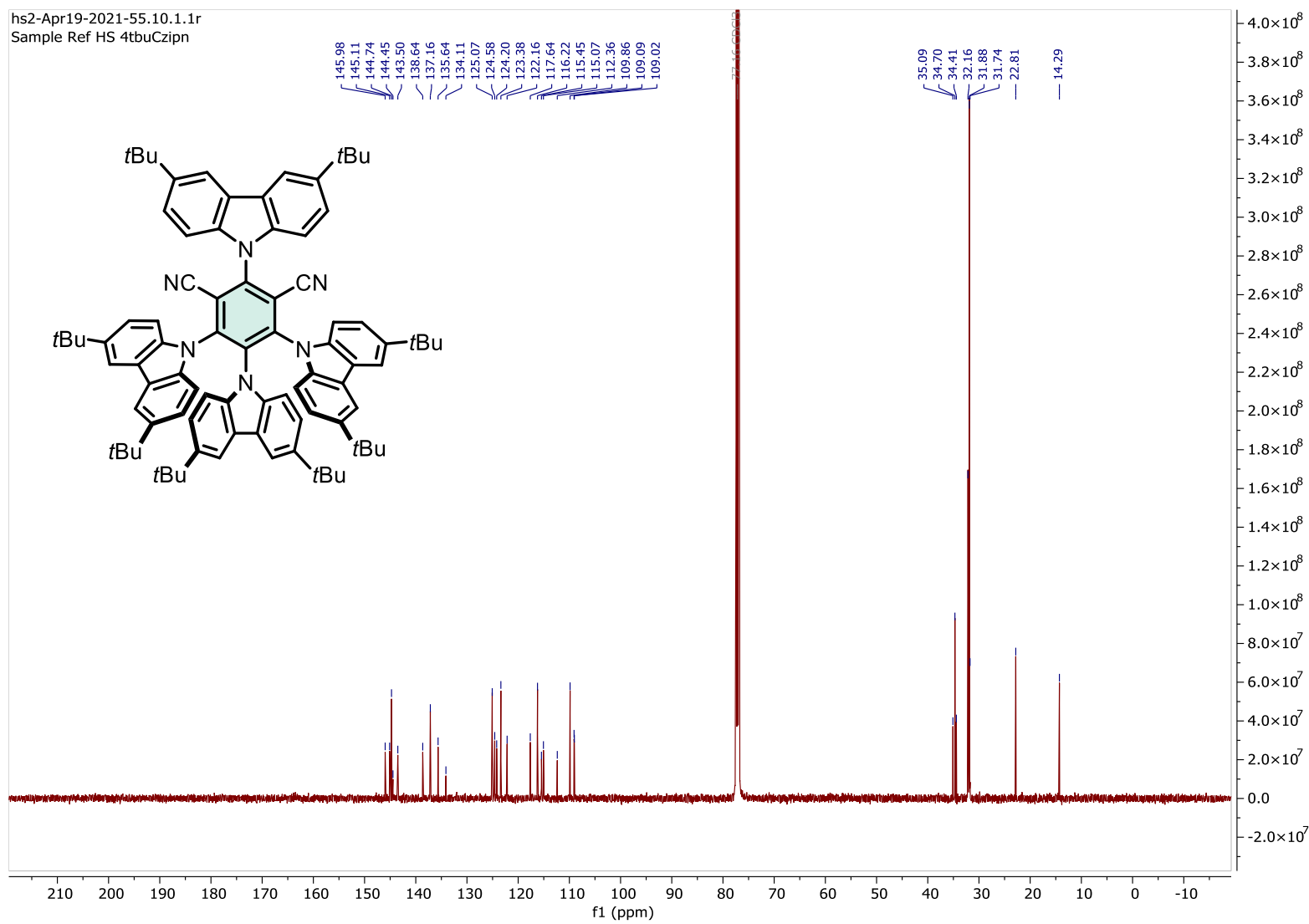


3f $-{ }^{1} \mathrm{H}$ NMR $\left(500 \mathrm{MHz}\right.$, Acetone- $\left.d_{6}\right)$

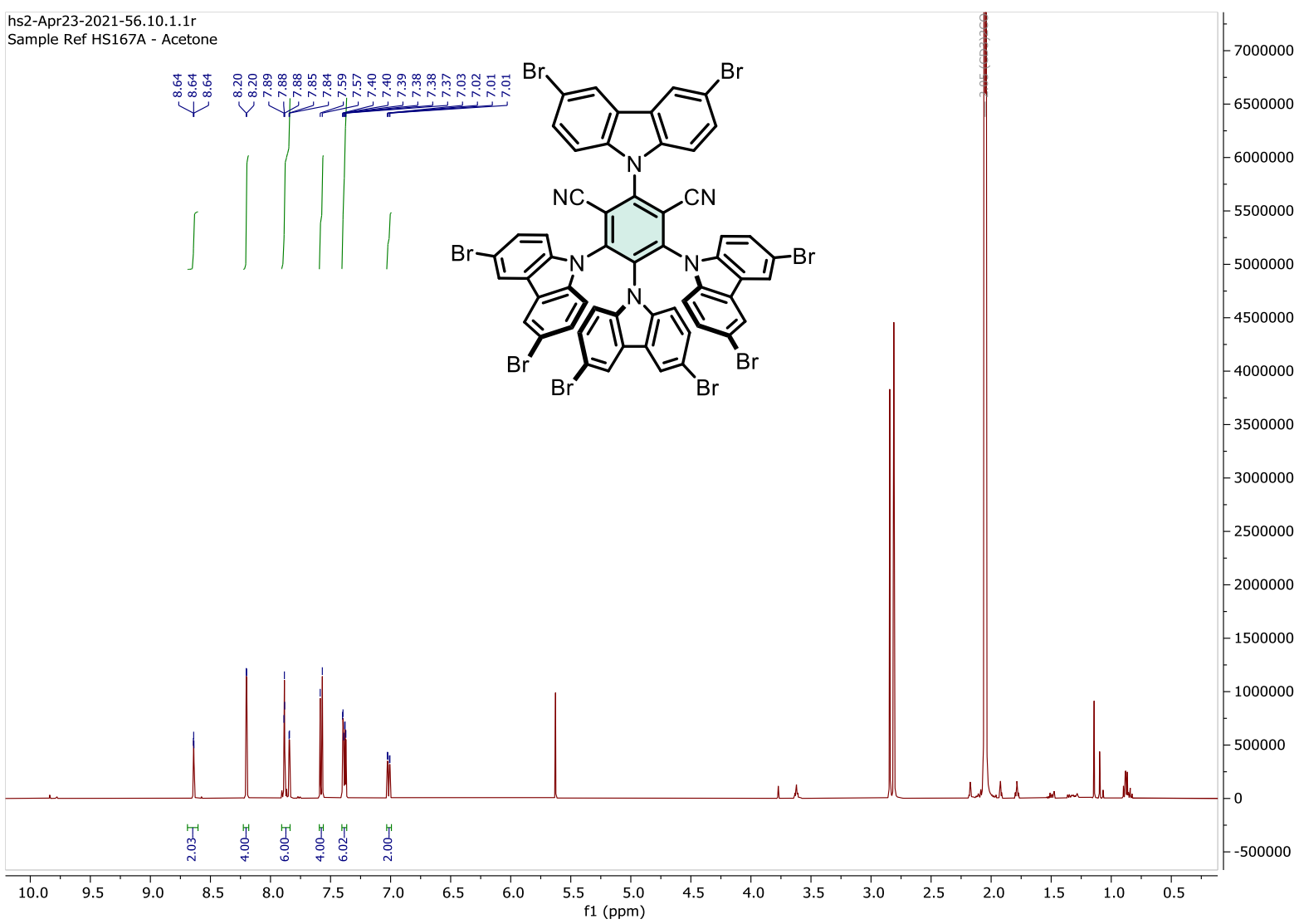

$3 \mathbf{f}-{ }^{13} \mathrm{C}$ NMR $\left\{{ }^{1} \mathrm{H}\right\}\left(126 \mathrm{MHz}\right.$, Acetone- $\left.d_{6}\right)$

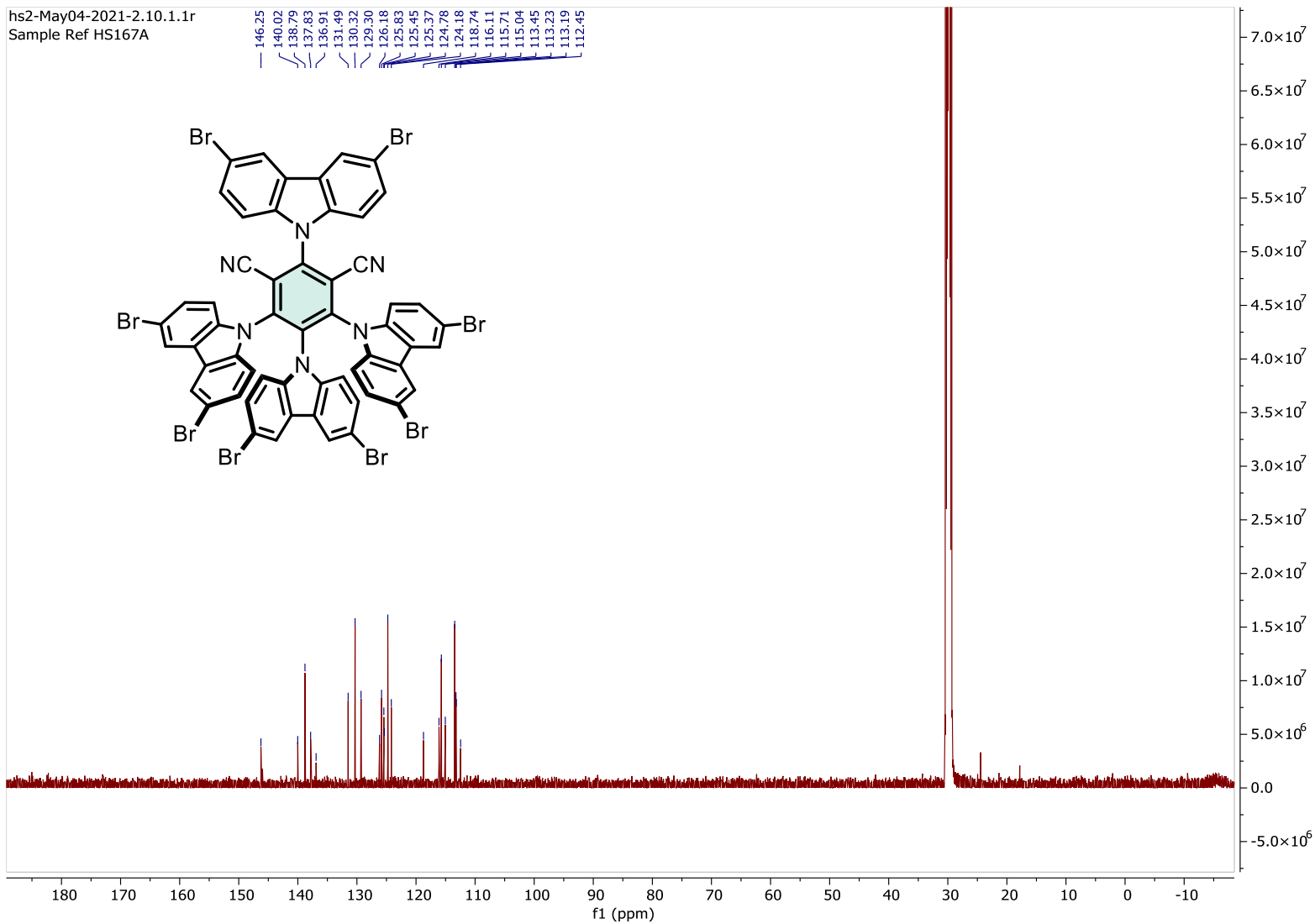


3g $-{ }^{1} \mathrm{H}$ NMR $\left(400 \mathrm{MHz}, \mathrm{CD}_{2} \mathrm{Cl}_{2}\right)$.

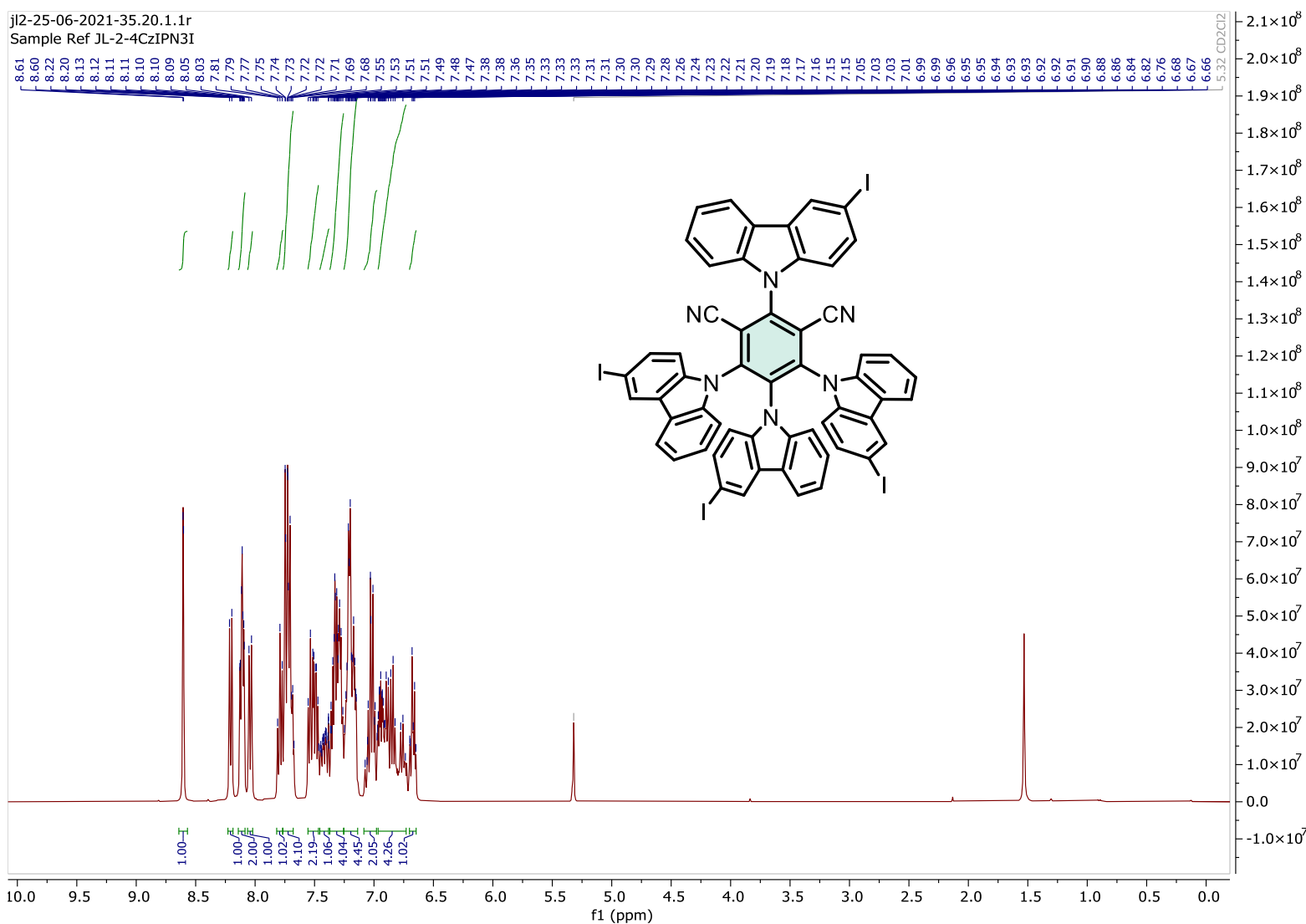

$3 \mathbf{g}-{ }^{13} \mathrm{C}\left\{{ }^{1} \mathrm{H}\right\}$ NMR $\left(101 \mathrm{MHz}, \mathrm{CD}_{2} \mathrm{Cl}_{2}\right)$
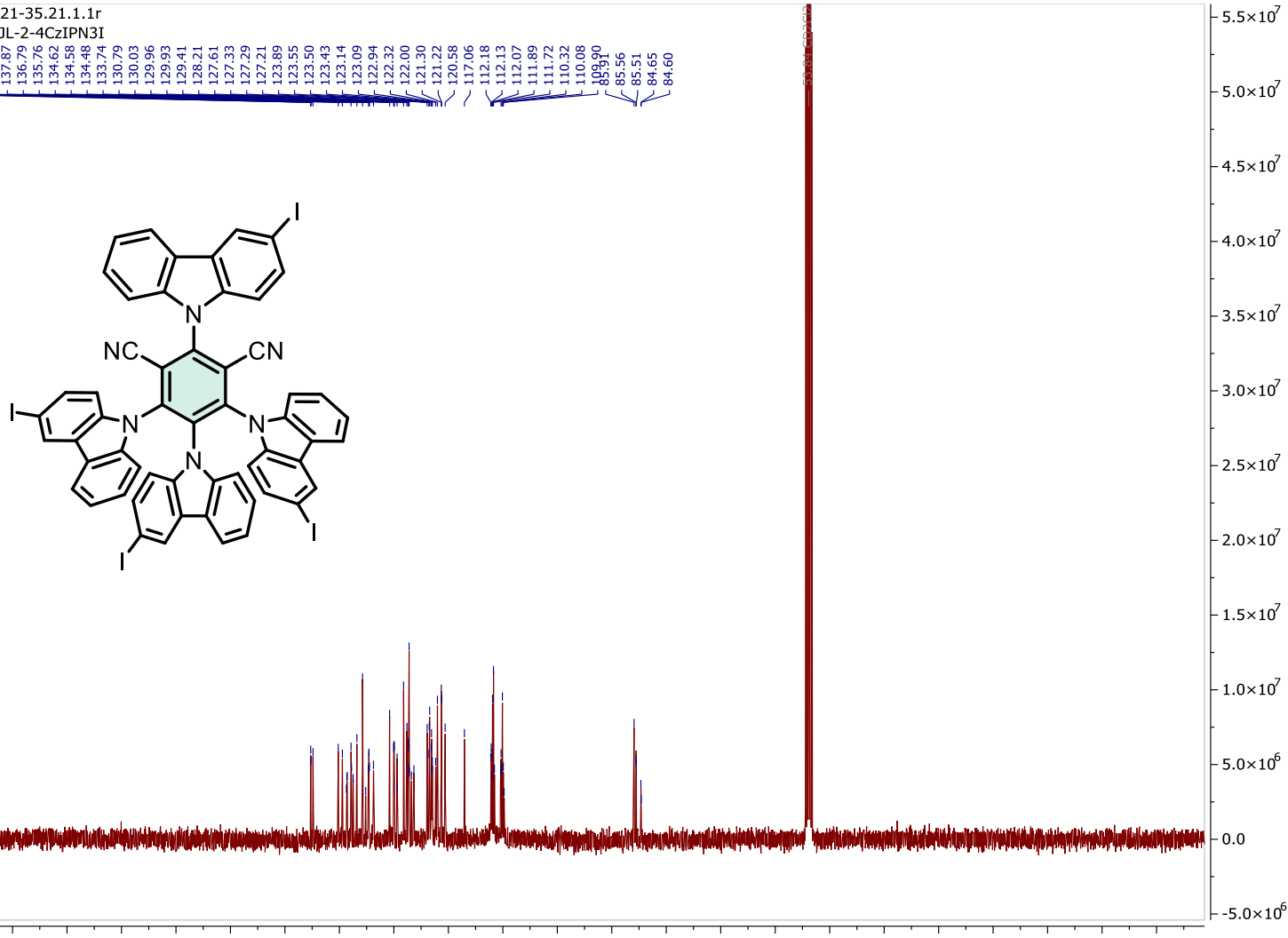

$\begin{array}{lllllllllll}210 & 200 & 190 & 180 & 170 & 160 & 150 & 140 & 130 & 120 & 110 \begin{array}{c}100 \\ \mathrm{f} 1(\mathrm{ppm})\end{array}\end{array}$ 